\title{
Maintaining Neonatal Normothermia during WHO Recommended Skin-to-Skin Contact in the Setting of Cesarean Section under Regional Anesthesia
}

\author{
Silvia Stirparo ${ }^{1}$, Alessio Farcomeni ${ }^{2}$, Alessandro Laudani ${ }^{1}$, Giorgio Capogna ${ }^{1}$ \\ ${ }^{1}$ Department of Anesthesiology, Città di Roma Hospital, Rome, Italy; ${ }^{2}$ Department of Experimental Medicine, La Sapienza Uni- \\ versity, Rome, Italy. \\ Email: silvia.stirparo@yahoo.it
}

Received February 22 ${ }^{\text {nd }}, 2013$; revised March $30^{\text {th }}, 2013$; accepted April $15^{\text {th }}, 2013$

Copyright (C) 2013 Silvia Stirparo et al. This is an open access article distributed under the Creative Commons Attribution License, which permits unrestricted use, distribution, and reproduction in any medium, provided the original work is properly cited.

\begin{abstract}
This study compared mothers' and newborns' temperatures $(\mathrm{T})$ when the WHO recommended skin-to-skin contact (SSC) was practiced during cesarean section under regional anesthesia. 139 neonates were randomized to be left in their mothers' arms warmed by a forced air warmer (SSC-FAW) or put in an incubator. Maternal and newborn rectal T was recorded immediately after birth, at 5, 10 and 15 minute intervals. Maternal and neonatal $\mathrm{T}$ was comparable between the groups. FAW is as effective as an incubator in preventing neonatal hypothermia while the mother is undergoing surgery in the operating room, while favouring SSC.
\end{abstract}

Keywords: Cesarean Section; Newborn; Temperature

\section{Introduction}

In the past the very act of holding the baby, newly born and still undressed, on the chest of the mother was essential to the survival of the newborn, and to this day many mothers still continue this practice. However, it is the norm in most industrialised societies to give birth in hospital, disrupting this instinctive gesture by having the baby taken away and clothed prior to being given back to their mothers.

One can imagine that this period of separation is particularly extended in the case of cesarean section, essentially an operation performed in cooled, sterile surroundings. These disruptions of early mother-infant interactions may in reality have a detrimental effect.

The WHO recommends maternal-neonatal skin-to-skin contact (SSC) for all newborns, regardless of their clinical condition, birth weight and gestational age [1].

This intervention may be a critical component for successful breastfeeding initiation, favours early motherchild bonding and possibly also has positive effects on excessive infant crying. It may promote reduced cardiopulmonary instability [2], and as far as the research shows is safe both in the short and long term.

It is not unusual for some neonates to experience moderate hypothermia, that is skin temperature between $32^{\circ} \mathrm{C}$ and $36^{\circ} \mathrm{C}$, even when the room temperature is relatively high, for example, in the region of $30^{\circ} \mathrm{C}$.

This tendency towards hypothermia is particularly evident in the first 10 to 20 minutes after delivery [3]. Several well recognised adverse effects on the neonate (particularly preterm) due to prolonged cold stress include increased oxygen consumption, acidosis and hypoglycemia, and adverse hemodynamic changes.

Cesarean sections are carried out at the cooler operating room temperatures thus exposing neonates born in this manner to a higher risk of hypothermia, further potentiated by lower maternal baseline temperature due to regional anesthesia [4] and because of less degree of mobilization of sympathoadrenal system as compared to vaginally delivered infants [5].

Furthermore, in view of the rise in cesarean births, it appears reasonable to recommend further research on SSC after cesarean delivery.

Almost all elective cesarean deliveries at our Institution are performed under neuraxial anesthesia, fathers are allowed to stay with the mother during surgery and newborns, after the routine clinical examination and Apgar score assignment, are briefly given to their parents and eventually put in the incubator until the end of sur- 
gery, in order to maintain temperature. As a result mothers have the chance to initiate and maintain SSC and to choose their breastfeeding program only upon return to the ward. However, this again may interfere with organized "pre-feeding behaviour" (spontaneous sucking and rooting, leading to successful suckling in the newborn) which is optimally stimulated in the first hour after birth.

In order to let the mothers to have SSC immediately after delivery, we aimed to introduce the use of a forced air warmer to keep the newborn temperature in normal range during this procedure.

The aim of this study was to compare mothers' and newborns' temperatures when SSC was practiced using a forced air warmer (FAW) compared to routine care (neonatal incubator) immediately after birth, during elective cesarean section under regional anesthesia in healthy mothers and healthy, term babies.

\section{Methods}

The protocol was approved by the local ethics committee. We enrolled 160 healthy full term mothers undergoing elective planned, repeat cesarean section under regional anesthesia. Women who fulfilled these inclusion criteria were invited to participate in the study at 35 - 37 weeks gestation, and written informed consent was obtained at the time of their antenatal anesthesia consultation.

Immediately after cord clamping all neonates were put under a radiant heat source and received the standard general clinical assessment performed by the neonatologist who was blinded to the study group and which included weight assessment, cord $\mathrm{pH}$ evaluation, and completion of Apgar score.

All neonates with Apgar score $<7$ at the first minute, umbilical cord $\mathrm{pH}$ less than 7.20, newborn weight less than $2500 \mathrm{~g}$ were excluded from the study.

Remaining neonates were randomized to receive SSC with FAW or routine care by using a computer-generated list.

Neonates assigned to the routine care group were dried off, fitted with a cap, wrapped in a blanket and then were place in an incubator after being shown to their mothers. Neonates assigned to the SSC-FAW group received the same treatment and were placed in direct contact with their mother's skin by being positioned on her chest and wrapped in the FAW (Bair Hugger ${ }^{\circledR}$ blankets and 750 Warming Unit, Arizant).

Maternal and newborn temperature was recorded immediately after birth and thereafter at the time of neonatal placing on the mother or into the incubator (Time 0), and subsequently at 5, 10 and 15 minute intervals. Maternal temperature was also measured immediately before anesthesia.
Temperature was measured with a rectal probe (Datex Ohmeda ${ }^{\circledR} 400$ series adult or neonatal temperature probe which has an accuracy of \pm 0.2 ) and connected to a Datex Ohmeda Cardiocap Monitor. Room temperature, assessed by a standard laboratory thermometer, was also noted. Incubator temperature was set at $34^{\circ} \mathrm{C}$. We also measured the temperature of the area inside the FAW wrap which was, on average, $34^{\circ} \mathrm{C}$.

Based on a previous study [6] of temperatures in neonates when SSC was practiced two hours after cesarean section, on the ward, we hypothesized that a sample size of 68 subjects in each group guaranteed us a power of at least $80 \%$ to demonstrate a mean difference of temperature $\geq 0.1^{\circ} \mathrm{C}$ with a significance level set to $5 \%$. Means, mean differences, and standard deviations were calculated and statistical analysis was done using Student's t test for unpaired samples.

\section{Results}

Of the 160 mothers enrolled, 139 newborns met the inclusion criteria at birth and were allocated to randomization, followed up and analyzed.

There were no differences in the mothers' and newborns' main characteristics (data not shown) as per inclusion protocol.

No maternal or newborn complications were noted.

Mean room $\mathrm{T}$ was $22.5^{\circ} \mathrm{C}$ (SD 0.2). Temperatures of neonates from the SSC-FAW group were comparable with those of the control group and were all within the normal range (Table 1).

\section{Discussion}

This study shows that FAW is as effective as an incubator in preventing neonatal hypothermia while the newborn baby is on the mother's chest as she is undergoing surgery in the operating room, thus favouring very early SSC.

Timing and duration of SSC may largely vary from immediately after the delivery to anytime between 1 and 24 hours after birth, but as neonates tend to be more alert within the first two hours of life, the earlier mother-infant contact is initiated, the better. It's not uncommon for newborns to be returned to their mothers more than two hours after delivery, when they are physiologically less alert, and may interact less with their mothers. In short, an essential window of opportunity may be lost due to current accepted hospital practice for cesarean section.

There are major obstacles for successful early SSC during cesarean birth, including practical, hospital and departmental policies, safety standards and/or healthcare professionals' concerns. Indeed the major difficulty in promoting SSC is the potential for neonatal hypothermia; 
Table 1. Maternal and neonatal temperatures. Time 0 is the time of neonatal placing on the mother or into the incubator. Data are presented as mean (SD). Temperatures are in ${ }^{\circ} \mathrm{C}$.

\begin{tabular}{ccccccc}
\hline & Before birth & At birth & Time 0 & 5 min & 10 min & 15 min \\
\hline Mother & & & & & \\
\hline SSC-FAW $(\mathrm{n}=69)$ & $36.7(0.2)$ & $36.7(0.2)$ & $36.5(0.3)$ & $36.3(0.5)$ & $36.2(0.5)$ & $36.4(0.4)$ \\
Control $(\mathrm{n}=70)$ & $36.8(0.3)$ & $36.6(0.3)$ & $36.6(0.3)$ & $36.4(0.4)$ & $36.4(0.4)$ & $36.5(0.5)$ \\
\hline Neonate & & $37.2(0.2)$ & $37(0.3)$ & $36.4(0.4)$ & $36.4(0.3)$ & $36.6(0.3)$ \\
\hline SSC-FAW $(\mathrm{n}=69)$ & & $37.2(0.3)$ & $37(0.3)$ & $36.4(0.3)$ & $36.4(0.3)$ & $36.5(0.3)$ \\
Control $(\mathrm{n}=70)$ & & & & & & \\
\hline
\end{tabular}

this is of particular relevance since cesarean sections are usually performed at a relatively low operating room temperature.

A previous study [6] demonstrated that SSC does not enhance the possibility of neonatal hypothermia when practiced two hours after cesarean delivery, when the mother returned in her room.

The WHO recommendations underline the value of early SSC to help mothers start early breastfeeding, even during cesarean deliveries [1].

To the best of our knowledge this is the first study to demonstrate that it is possible to offer SSC to all healthy mothers and their babies undergoing elective cesarean section under regional anesthesia by using a FAW system to maintain newborn temperature while in their mother's arms.

However the aim of our study was to investigate the efficacy of FAW to maintain healthy newborn temperature, safely allowing very early SSC, and did not investigate the effects of such intra-operative early contact on breastfeeding initiation and early mother-infant attachment once the couple is on the ward. We are aware that maternal preference and local hospital policy should be taken into account, and that further investigation into this specific issue is needed. Nevertheless we believe that there is great benefit to be gained by not delaying in initiating early SSC and indeed further research may be directed at studying the viability and benefit of continuing this practice during transport and on return to the ward.

In conclusion, FAW may allow WHO SSC recommendations to be fulfilled during elective cesarean section under regional anesthesia in healthy mothers and healthy term babies, when the standard operating room temperature is unfavourable.

\section{REFERENCES}

[1] World Health Organization, Division of Child Health and Development, "Evidence for the Ten Steps to Successful Breastfeeding,” 1998.

[2] S. Carfoot, P. Williamson and R. Dickson, "A Systematic Review of Randomised Controlled Trials Evaluating the Effect of Mother/Baby Skin-To-Skin Care on Successful Breastfeeding," Midwifery, Vol. 19, No. 2, 2003, pp. 148155. doi:10.1016/S0266-6138(02)00102-X

[3] K. Hammarlund, G. E. Nilsson, P. A. Oberg and G. Sedin, "Transepidermal Water Loss in Newborn Infants: V. Evaporation from the Skin and Heat Exchange during the First Hours of Life,” Acta Paediatrica, Vol. 69, No. 3, 1980, pp. 385-392. doi:10.1111/j.1651-2227.1980.tb07097.x

[4] T. Matsukawa, D. I. Sessler, R. Christensen, et al., "Heat Flow and Distribution during Epidural Anesthesia,” Anesthesiology, Vol. 83, No. 5, 1995, pp. 961-967. doi:10.1097/00000542-199511000-00008

[5] K. Christensson, C. Siles, T. Cabrera, A. Belaustequi, P. de la Fuente, H. Lagercrantz, et al., "Lower Body Temperatures in Infants Delivered by Cesarean Section than in Vaginally Delivered Infants,” Acta Paediatrica, Vol. 82, No. 2, 1993, pp. 128-131. doi:10.1111/j.1651-2227.1993.tb12622.x

[6] S. Gouchon, D. Gregori, A. Picotto, G. Patrucco, M. Nangeroni and P. Di Giulio, "Skin-to-Skin Contact after Cesarean Delivery," An Experimental Study. Nursing Research, Vol. 59, No. 2, 2010, pp. 78-84. doi:10.1097/NNR.0b013e3181d1a8bc 